\title{
Consumer Attitudes on Salt and Fat Reduced Foods in the Republic of Ireland
}

\author{
Susann Fellendorf ${ }^{1,2}$, Joseph P. Kerry ${ }^{2 *}$, Maurice G. O'Sullivan ${ }^{1 *}$ \\ ${ }^{1}$ The Sensory Group, School of Food and Nutritional Sciences, University College Cork, Cork, Ireland \\ ${ }^{2}$ The Food Packaging Group, School of Food and Nutritional Sciences, University College Cork, Cork, Ireland \\ Email: *joe.kerry@ucc.ie, ^maurice.osullivan@ucc.ie
}

How to cite this paper: Fellendorf, S., Kerry, J.P. and O'Sullivan, M.G. (2018) Consumer Attitudes on Salt and Fat Reduced Foods in the Republic of Ireland. Food and Nutrition Sciences, 9, 880-898. https://doi.org/10.4236/fns.2018.97066

Received: April 20, 2018

Accepted: July 23, 2018

Published: July 26, 2018

Copyright (c) 2018 by authors and Scientific Research Publishing Inc. This work is licensed under the Creative Commons Attribution International License (CC BY 4.0).

http://creativecommons.org/licenses/by/4.0/

cc) (7) Open Access

\begin{abstract}
Public health campaigns are continually promoting healthier foods and in parallel many food manufacturers are working on recipes low in fat and salt. The following survey investigates how well consumers $(n=1045)$ in Ireland have assimilated the message of consuming healthier foods, including salt and fat reduced foods, into their diet. It seems that campaigns regarding the importance of a balanced diet have generally been adopted by respondents, as only a minority of the participants did not take care of their diet, independent of age, gender and level of education. More than half of the participants carefully maintained a balanced diet. Furthermore, an increase in purchasing salt reduced food was observed, though fat reduced food products were already better accepted. In general, men, young adults ( $<30$ years) and lower educated people purchased less salt and fat reduced foods and took less care in maintaining a balanced diet. No conflict was observed for traditional foods reduced in salt and fat content when their intrinsic sensory character is maintained. The majority of the participants were aware of the health risks of a diet rich in salt and fat, though less participants transferred this knowledge into their diet. A very high demand for more salt and fat reduced foods on the market was observed although the smaller range of salt and fat reduced products and their higher prices might diminish the level of consumer acceptance. Launching taxation and subsidies in tandem might counteract this and furthermore, user-friendly product labelling schemes are required.
\end{abstract}

\section{Keywords}

Survey, Reduced Salt, Fat, Attitudes

\section{Introduction}

\subsection{Background}

Changing consumer lifestyle patterns show a significant impact on consumer di- 
ets. A greater number of women are at work, people work longer hours and spend more time on leisure activities [1], which has caused an increased demand for convenience foods, with most energy dense and poor in nutrition. With the increased consumption of processed foods, salt intake has increased to a level of 9 to $12 \mathrm{~g}$ salt per day in nearly all countries worldwide [2], which is 40 to 50 times higher than the evolutionary intake ( $0.25 \mathrm{~g}$ salt per day) [3]. A consistent linear relationship between salt intake, blood pressure levels and risk of coronary heart disease (CVD) and stroke are thus evident [4].

Due to the higher consumption of processed foods high in saturated fatty acids, the prevalence of obesity has increased severely in most countries [5]. The dietary saturated fatty acids raise the level of serum low-density lipoprotein (LDL)-cholesterol, an identified risk factor for cardiovascular disease [6].

People of higher socio-economic status consume a greater variety of food products with a higher nutritional value [7]. In contrast, the diet of the lower socio-economic group comes from food cheap in energy [8]. Therefore, the occurrence of hypertension, stroke, heart disease and cancer are higher in this group.

Consequently, the World Health Organization (WHO) called on all countries to reduce daily salt consumption to less than $5 \mathrm{~g} /$ day with the aim of reducing salt intake by $30 \%$ by 2025 [9] and suggest a total fat intake between $15 \%$ and $30 \%$ of the dietary energy and a daily intake of polyunsaturated fatty acids (PUFAs) between $6 \%$ and $11 \%$ based on daily energy intake [10].

Food manufacturers have to achieve satisfactory sensory quality in reduced salt and fat products, which is challenging as salt and fat performs multi-purpose roles in many processed foods and beverages. Salt is a preservative and imparts not only a salty taste, but also enhances flavour, inhibits bitterness [11] and is important for yield in processed meats due to its water binding functionality [12]. The component fat contributes to food texture which is highly product specific [13], plays a role in [14] and in carrying aroma as well as satiety [15]. The food industry has already successfully started to implement processed foods low in negative ingredients such as salt, fat and sugar [16]-[25]. Different strategies have been attempted to achieve this objective including: reducing the total amount of salt or by (partly) substitution of sodium chloride with salt replacer ingredients [26]-[41]. Traditional foods show an insufficient adoption of these trends, possibly due the resistance to reformulate because of conservatism [42], which has to be overcome. Various sensory methods combining hedonic and descriptive components, with some using novel methods, have also been employed by some researchers for the nutrient and flavour optimisation of processed foods and beverages [43]-[52].

Dietary reductions in salt and fat intake are progressed by raising consumers' awareness. Education through public health campaigns (via television, radio, newspapers, brochures, internet, social media and press releases) focuses on health risks leading consumers to purchase healthier food products, encouraging cooking from raw ingredients and for them to use less salt and fat during cooking and at the table [53]. 
Many countries have begun with campaigns to reduce the dietary salt and fat consumption in their population. The demand for healthier food has already increased. Hence, consumers demand for healthier and more natural food is confronted with the need for also convenience foods [54].

\subsection{Framework of the Present Study}

Many food manufacturers are working on recipes low in fat and salt, while in parallel public health campaigns are promoting the consumption of healthier foods. The following survey investigates how well consumers have adopted the message of consuming healthier foods, including salt and fat reduced foods, into their diet.

It is of great interest to determine if consumers are aware of the health risks caused from a diet rich in salt and fat, and furthermore if they utilize a balanced diet and buy salt and fat reduced foods. The present study will also clarify if there is a conflict between innovation and traditional food, specifically traditional white and black puddings. White and black puddings have a very high value for the Irish and British consumer since they are particularly a special feature of the traditional Irish and English breakfast with producers closely guarding their recipes.

Furthermore, product development of salt and fat reduced foods involves the use of higher quality ingredients and/or replacers which leads to cost increases. The survey will determine if consumers' disposable income generally inhibits spending more money on foods and if consumers are willing to pay more for healthier foods. Finally, the present study will give an update if a demand for further salt and fat reduced food products on the market still exists.

\section{Methodology}

\subsection{Participants and Procedure}

1045 consumers took part in the online survey titled "Salt and fat reduced foods" as part of the PROSSLOW (2017) [55] project [12] [29] [30] [31] [32] [40] [41], which completed in October 2017. The survey was distributed to a broad range of individuals living in Ireland (Table 1) and circulated through email and social media. The survey was conducted in English and lasted about 10 minutes. To reach a high number of participation the survey was short with 16 questions. All answer possibilities were listed (except the question on nationality).

\subsection{Questionnaire}

The questionnaire was divided into four sections. The first part contained demographic questions, followed by questions on financial situation. The third part was about participants' consumption characteristics with the focus on salt and fat reduced foods and the last section presented questions on knowledge and beliefs.

\subsubsection{Questions on Demographic}

The participants were asked four demographic questions: age, gender, national- 
ity and level of education. Age was class-divided as follow: <20, 20 - 29, 30 - 39, 40 - 49, 50 - 59 and $\geq 60$ and level of education was subdivided in to five categories and listed from the lowest to the highest level: no school certificate, leaving certificate, primary degree and diploma, postgraduate degree and higher degree. The chosen categories based on the Irish national framework of qualification (Quality and Qualifications Ireland, 2003). In Ireland the "leaving certificate" is gained by passing successfully school exams at second level. The category "primary and diploma" covers the higher and advanced certificates, bachelor and diploma degrees. Furthermore, education level "postgraduate" comprises master degree and postgraduate diploma. The highest education category "higher degree" contents doctoral degree and higher doctorate.

\subsubsection{Questions on Financial Situation}

Since expendable income influences consumer purchase behaviour, the second part of the questionnaire included two questions based on income and food purchase: "What percent of your income do you spend on food per month?" and followed by "Would you spend more money on food, if you had more money available?"

\subsubsection{Questions on Consumption Characteristics}

To explore participants' food habits three questions were presented: "Do you consume a balanced diet (vegetables, fruits, fibres, bread, meat, dairy products)?", "Do you purchase salt reduced food?" and "Do you purchase fat reduced food?". It was assumed that the participants had a basic knowledge regarding maintaining balanced diet, nevertheless the components of a balanced diet were listed in brackets.

Questions on specific consumption of products then followed. Beginning with: "Would you eat salt and fat reduced white and black pudding sausages?". Three answer choices were given as follows: "Yes, even when the taste is different to conventional white and black pudding sausages", Yes, if the taste is similar to conventional white and black pudding sausages" or "Not at all". White and black pudding sausages are popular in Ireland and in the United Kingdom and contribute particularly a special feature of the traditional Irish breakfast. It is not just eaten at home by the domestic population, Bed \& Breakfasts and Hotels traditionally serve these products to tourists. Therefore, white and black pudding sausages have a high local value which is the reason they were chosen as a representative of traditional foods. The second question was a decision question: "Would you purchase salt and fat reduced food even when the food is more expensive?" Only, if the participants respond with "Yes" they were then asked "If yes, how much more money in percentage would you spend on salt and fat reduced foods?".

\subsubsection{Questions on Knowledge and Beliefs}

In the last part of the questionnaire participants were asked about their knowledge and beliefs: "Do you know that high sodium levels in food can cause hy- 
pertension, cardiovascular diseases and stroke?", "Do you know that high saturated fatty acids level in food can cause obesity, diabetes and stroke?", "Do you think there is a greater need in informing people about the impact of sodium and saturated fatty acids in food on body health?" and "Do you think there is a greater need for salt and fat reduced products on the market?". All four questions are decision questions.

\subsection{Data Analyses}

IBM SPSS Statistics 20 software package (SPSS, Chicago, IL, USA) was used to carry out the statistical analyses. Assuming a normal distribution of the values, the Bonferroni method was performed in order to evaluate the presence of significant differences between age and level of education [56]. Furthermore, Chi-Square analyses combined with post-hoc testing was used in order to evaluate significant differences in gender [57].

\section{Results}

\subsection{Demographic-Results}

The survey, "Salt and fat reduced foods" completed 1045 participants, with 71\% female and $29 \%$ male (Table 1 ). In total, $96 \%$ of the participants were European,

Table 1. Demographic.

\begin{tabular}{|c|c|c|c|c|c|}
\hline \multicolumn{6}{|c|}{ Age [\%] } \\
\hline$<20$ & $20-29$ & $30-39$ & $40-49$ & $50-59$ & $\geq 60$ \\
\hline 19.6 & 34.5 & 14.5 & 16.4 & 10.2 & 4.8 \\
\hline \multicolumn{6}{|c|}{ Gender [\%] } \\
\hline female & male & & & & \\
\hline 71.1 & 28.9 & & & & \\
\hline \multicolumn{6}{|c|}{ Education [\%] } \\
\hline No school certificate & $\begin{array}{l}\text { Leaving } \\
\text { certificate }\end{array}$ & $\begin{array}{c}\text { Primary degree + } \\
\text { Diploma }\end{array}$ & $\begin{array}{c}\text { Post- } \\
\text { graduate }\end{array}$ & $\begin{array}{l}\text { Higher } \\
\text { degree }\end{array}$ & \\
\hline 0.8 & 39.1 & 23.3 & 19.7 & 17.1 & \\
\hline \multicolumn{6}{|c|}{ Nationality [\%] } \\
\hline European & & American & Asian & African & Australien \\
\hline 96.4 & & 1.5 & 1.2 & 0.7 & 0.2 \\
\hline Irish & 87.7 & & & & \\
\hline UK & 2.9 & & & & \\
\hline German & 1.5 & & & & \\
\hline French & 0.8 & & & & \\
\hline Dutch & 0.4 & & & & \\
\hline Spanish & 0.4 & & & & \\
\hline other & 2.7 & & & & \\
\hline
\end{tabular}


of these $88 \%$ Irish, 3\% British, 2\% German, 1\% Spanish and other nationality less than $1 \%$ (Table 1 ). The greatest percentage of respondents $(34.5 \%)$ were aged 20 - 29, followed by those aged under 20 (19.6\%), $40-49$ (16.4\%) and $30-$ $39(14.5 \%)$. Adults aged 50 to 59 took part with $10.2 \%$ and adults aged 60 and older accounted 4.8\% (Table 1). "No school certificate" individuals accounted for only $0.8 \%$ of participants. A total of $39.1 \%$ of participants were educated to "leaving certificate" and $23.3 \%$ to "primary degree and diploma". The higher levels "postgraduate" and "higher degree" were evenly distributed with $19.7 \%$ and accordingly $17.1 \%$ (Table 1 ).

\subsection{Financial Situation-Results}

As shown in Table 2, two-thirds of participants spend between $15 \%$ and $30 \%$ of their income on food. The most responses with $23 \%$ were achieved for $20 \%$ of income spend on foods. For this, no significant differences were found in age, gender and level of education. Furthermore, only $4 \%$ of participants spend $5 \%$ of income on groceries. Data analysis revealed that no significant differences existed in gender, across the various age groups and levels of education.. Fifteen percent of the participants spend $40 \%$ and more of their income on food. No statistical differences in age and gender were found and no definitive pattern was shown for different educational levels.

Fifty percent of the participants responded to the question "Would you spend more money on food, if you had more money available?" with "yes" and 50\%

Table 2. Financial aspects.

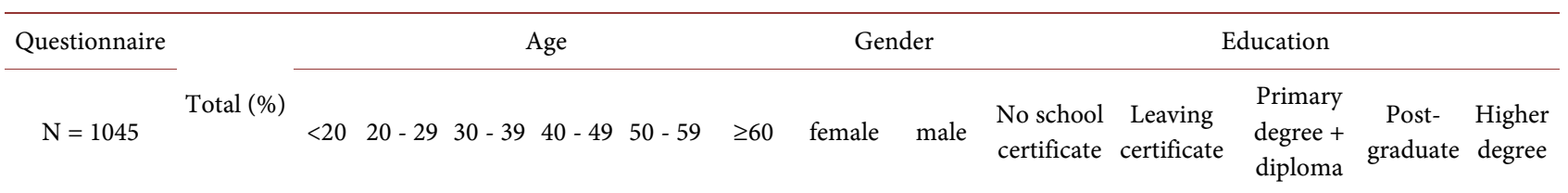

How many percent of your net income do you approximately spend on food per month?

\begin{tabular}{|c|c|c|c|c|c|c|c|c|c|c|c|c|c|c|}
\hline 5 & 4 & $10.2^{\mathrm{a}}$ & $2.8^{\mathrm{b}}$ & $2.6^{\mathrm{a}, \mathrm{b}}$ & $2.3^{\mathrm{b}}$ & $0.9^{\mathrm{b}}$ & $2.0^{\mathrm{a}, \mathrm{b}}$ & $4.3^{\mathrm{a}}$ & $3.0^{\mathrm{a}}$ & $0.0^{\mathrm{a}, \mathrm{b}}$ & $6.8^{\mathrm{a}}$ & $2.5^{\mathrm{a}, \mathrm{b}}$ & $2.4^{\mathrm{a}, \mathrm{b}}$ & $1.1^{\mathrm{b}}$ \\
\hline 10 & 9 & $7.8^{\mathrm{a}}$ & $7.5^{\mathrm{a}}$ & $11.8^{\mathrm{a}}$ & $13.5^{\mathrm{a}}$ & $11.2^{\mathrm{a}}$ & $6.2^{\mathrm{a}}$ & $9.6^{\mathrm{a}}$ & $9.3^{\mathrm{a}}$ & $28.6^{\mathrm{a}, \mathrm{b}}$ & $6.1^{\mathrm{a}}$ & $7.8^{\mathrm{a}, \mathrm{b}}$ & $11.7^{\mathrm{a}, \mathrm{b}}$ & $16.2^{\mathrm{b}}$ \\
\hline 15 & 15 & $7.3^{\mathrm{a}}$ & $13.6^{\mathrm{a}, \mathrm{c}}$ & $27.0^{\mathrm{b}}$ & $16.4^{\mathrm{a}, \mathrm{b}, \mathrm{c}}$ & $18.7^{\mathrm{b}, \mathrm{c}}$ & $16.3^{\mathrm{a}, \mathrm{b}, \mathrm{c}}$ & $14.9^{\mathrm{a}}$ & $16.6^{\mathrm{a}}$ & $0.0^{\mathrm{a}, \mathrm{b}}$ & $7.8^{\mathrm{b}}$ & $18.0^{\mathrm{a}}$ & $22.8^{\mathrm{a}}$ & $21.2^{\mathrm{a}}$ \\
\hline 20 & 23 & $20.5^{\mathrm{a}}$ & $22.2^{\mathrm{a}}$ & $25.7^{\mathrm{a}}$ & $22.8^{\mathrm{a}}$ & $24.3^{\mathrm{a}}$ & $28.6^{\mathrm{a}}$ & $22.2^{\mathrm{a}}$ & $24.8^{\mathrm{a}}$ & $14.3^{\mathrm{a}}$ & $22.5^{\mathrm{a}}$ & $23.4^{\mathrm{a}}$ & $23.3^{\mathrm{a}}$ & $23.5^{\mathrm{a}}$ \\
\hline 25 & 13 & $11.7^{\mathrm{a}}$ & $14.7^{\mathrm{a}}$ & $9.2^{\mathrm{a}}$ & $10.5^{\mathrm{a}}$ & $15.9^{\mathrm{a}}$ & $12.2^{\mathrm{a}}$ & $12.5^{\mathrm{a}}$ & $12.9^{\mathrm{a}}$ & $14.3^{\mathrm{a}}$ & $10.8^{\mathrm{a}}$ & $15.2^{\mathrm{a}}$ & $13.1^{\mathrm{a}}$ & $12.8^{\mathrm{a}}$ \\
\hline 30 & 14 & $13.2^{\mathrm{a}}$ & $15.8^{\mathrm{a}}$ & $10.5^{\mathrm{a}}$ & $15.2^{\mathrm{a}}$ & $12.1^{\mathrm{a}}$ & $10.2^{\mathrm{a}}$ & $13.2^{\mathrm{a}}$ & $15.2^{\mathrm{a}}$ & $0.0^{\mathrm{a}}$ & $14.9^{\mathrm{a}}$ & $13.9^{\mathrm{a}}$ & $14.1^{\mathrm{a}}$ & $11.2^{\mathrm{a}}$ \\
\hline 35 & 7 & $7.8^{\mathrm{a}}$ & $6.4^{\mathrm{a}}$ & $5.3^{\mathrm{a}}$ & $8.8^{\mathrm{a}}$ & $1.9^{\mathrm{a}}$ & $10.2^{\mathrm{a}}$ & $7.4^{\mathrm{a}}$ & $4.6^{\mathrm{a}}$ & $14.3^{\mathrm{a}}$ & $7.6^{\mathrm{a}}$ & $7.0^{\mathrm{a}}$ & $5.8^{\mathrm{a}}$ & $4.5^{\mathrm{a}}$ \\
\hline 40 & 9 & $11.7^{\mathrm{a}}$ & $11.1^{\mathrm{a}}$ & $4.6^{\mathrm{a}}$ & $6.4^{\mathrm{a}}$ & $11.2^{\mathrm{a}}$ & $6.1^{\mathrm{a}}$ & $10.1^{\mathrm{a}}$ & $7.3^{\mathrm{a}}$ & $0.0^{\mathrm{a}, \mathrm{b}, \mathrm{c}}$ & $13.9^{\mathrm{b}}$ & $7.4^{\mathrm{a}, \mathrm{b}, \mathrm{c}}$ & $6.3^{\mathrm{a}, \mathrm{c}}$ & $5.0^{c}$ \\
\hline$>40$ & 6 & $9.8^{\mathrm{a}}$ & $6.1^{\mathrm{a}}$ & $3.3^{\mathrm{a}}$ & $4.1^{\mathrm{a}}$ & $3.7^{\mathrm{a}}$ & $8.2^{\mathrm{a}}$ & $5.8^{\mathrm{a}}$ & $6.3^{\mathrm{a}}$ & $28.6^{\mathrm{b}}$ & $9.5^{\mathrm{b}}$ & $4.9^{\mathrm{a}, \mathrm{b}}$ & $0.5^{\mathrm{a}}$ & $4.5^{\mathrm{a}, \mathrm{b}}$ \\
\hline \multicolumn{15}{|c|}{ Would you spend more money on food, if you had more money available? } \\
\hline Yes & 50 & $53.2^{\mathrm{a}}$ & $57.6^{\mathrm{a}}$ & $50.7^{\mathrm{a}, \mathrm{c}}$ & $46.8^{\mathrm{a}, \mathrm{c}}$ & $32.7^{\mathrm{b}, \mathrm{c}}$ & $18.4^{\mathrm{b}}$ & $51.7^{\mathrm{a}}$ & $44.4^{\mathrm{a}}$ & $85.7^{\mathrm{a}, \mathrm{b}, \mathrm{c}}$ & $55.7^{\mathrm{b}}$ & $52.9^{\mathrm{a}, \mathrm{b}}$ & $43.2^{\mathrm{a}, \mathrm{c}}$ & $36.9^{c}$ \\
\hline No & 50 & $46.8^{\mathrm{a}}$ & $42.4^{\mathrm{a}}$ & $49.3^{\mathrm{a}, \mathrm{c}}$ & $53.2^{\mathrm{a}, \mathrm{c}}$ & $67.3^{\mathrm{b}, \mathrm{c}}$ & $81.6^{\mathrm{b}}$ & $48.3^{\mathrm{a}}$ & $55.6^{\mathrm{a}}$ & $14.3^{\mathrm{a}, \mathrm{b}, \mathrm{c}}$ & $44.3^{\mathrm{b}}$ & $47.1^{\mathrm{a}, \mathrm{b}}$ & $56.8^{\mathrm{a}, \mathrm{c}}$ & $63.1^{\mathrm{c}}$ \\
\hline
\end{tabular}

a,b,c Percentages within question and row sharing different letters are significantly different. 
with "no" (Table 2). With increasing age and with a higher level of education significant less people answered "yes". Only $33 \%$ percent in the age group of 50 to 59 and $18 \%$ in the age group over 59 would like to spend more money on food. Again, no significant differences in gender were observed.

\subsection{Consumption Characteristics-Results}

"Do you take care of a balanced diet?" answered 59.2\% "Yes, completely", 38.4\% "Just a bit" and only $2.4 \%$ "Not at all" (Table 3 ). With increasing age and level of education, significantly more people voted "Yes, completely". Furthermore, significantly more women take care of a complete balanced diet than men. Adults aged under 20, and the age group 20 to 29 accounted for $52 \%$ and $44 \%$, respectively, for "Just a bit". For the response "Not at all" no statistical differences in attitudes between age and gender were found, and no definitive pattern was shown for different educational levels.

In total, $43 \%$ of the participants purchase salt reduced foods and almost two-thirds purchase fat reduced foods (Table 3). Without any exception, more fat than salt reduced foods were purchased independent of age, gender and level of education. Adults aged under 20 reached the significant lowest purchase rate with $29 \%$ for salt reduced foods. Significantly more women purchase salt (48\%) and fat $(70 \%)$ reduced groceries than men ( $32 \%$ and $50 \%$, respectively). Participants with "primary and diploma" degrees significantly accounted more for purchasing salt and fat reduced foods than participants with just the "leaving certificate", though no definitive pattern was observed for the various levels of education.

"Would you purchase salt and fat reduced white and black pudding" responded half of the participants with "Yes, if the taste is similar to conventional white and black pudding sausages" and one-third with "Not at all" (Table 3). Only 15\% would even purchase salt and fat reduced white and black pudding with a different taste to conventional white and black pudding sausages. In general, no particular age group and gender was found to vote statistically more or less than the others. Furthermore, no pattern was observed for different educational levels.

Data analysis revealed that $56 \%$ of participants would purchase salt and fat reduced foods even when they are more expensive (Table 3). Moreover, significantly more adults aged over 29 and more women (than men) would spend more money on salt and fat reduced foods. Although, participants with a school leaving certificate voted significantly less for paying more money on salt and fat reduced foods. However, in total $33 \%$ and accordingly $16 \%$ of all participants would spend $5 \%$ to $15 \%$ and $15 \%$ to $30 \%$, respectively, more money on salt and fat reduced foods (Table 3). Thus, statistical differences were observed across the various age groups and levels of education, though no definitive patterns were evident.

\subsection{Knowledge and Beliefs-Results}

Two questions were asked on respondents' knowledge. In total, $92 \%$ of the 
Table 3. Consumption characteristics.

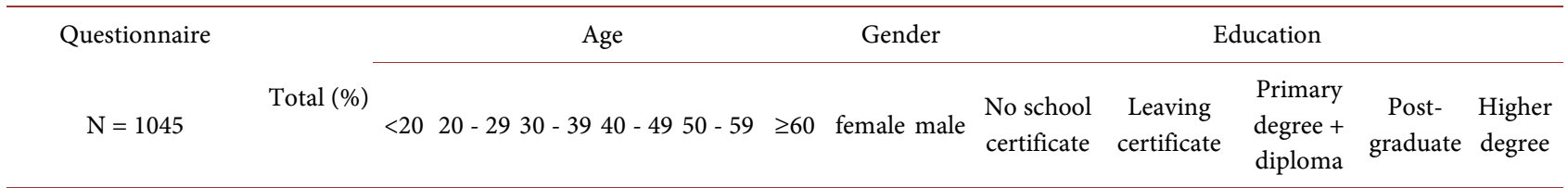

Do you take care about a balanced diet (vegetables, fruits, fibers, bread, meat, dairy products)?

\begin{tabular}{cccccccccccccccc}
\hline Yes, completely & 59 & $43.4^{\mathrm{a}}$ & $53.2^{\mathrm{a}}$ & $68.4^{\mathrm{b}}$ & $67.8^{\mathrm{b}}$ & $72.9^{\mathrm{b}}$ & $81.6^{\mathrm{b}}$ & $63.0^{\mathrm{a}}$ & $50.0^{\mathrm{b}}$ & $57.1^{\mathrm{a}, \mathrm{b}, \mathrm{c}}$ & $47.9^{\mathrm{c}}$ & $59.5^{\mathrm{a}}$ & $67.0^{\mathrm{a}, \mathrm{b}}$ & $76.0^{\mathrm{b}}$ \\
Just a bit & 38 & $51.7^{\mathrm{a}}$ & $44.3^{\mathrm{a}, \mathrm{c}}$ & $29.6^{\mathrm{b}}$ & $31.6^{\mathrm{b}, \mathrm{c}}$ & $26.2^{\mathrm{b}}$ & $16.3^{\mathrm{b}}$ & $34.9^{\mathrm{a}}$ & $47.0^{\mathrm{b}}$ & $28.6^{\mathrm{a}, \mathrm{b}, \mathrm{c}}$ & $48.2^{\mathrm{b}}$ & $38.9^{\mathrm{a}, \mathrm{b}}$ & $31.6^{\mathrm{a}, \mathrm{c}}$ & $23.4^{\mathrm{c}}$ \\
Not at all & 2 & $4.9^{\mathrm{a}}$ & $2.5^{\mathrm{a}}$ & $2.0^{\mathrm{a}}$ & $0.6^{\mathrm{a}}$ & $0.9^{\mathrm{a}}$ & $2.0^{\mathrm{a}}$ & $2.1^{\mathrm{a}}$ & $3.0^{\mathrm{a}}$ & $14.3^{\mathrm{a}}$ & $3.9^{\mathrm{a}, \mathrm{b}}$ & $1.6^{\mathrm{a}, \mathrm{b}}$ & $1.4^{\mathrm{a}, \mathrm{b}}$ & $0.6^{\mathrm{b}}$ \\
\hline
\end{tabular}

Do you purchase salt reduced food?

\begin{tabular}{|c|c|c|c|c|c|c|c|c|c|c|c|c|c|c|}
\hline Yes & 43 & $29.3^{\mathrm{a}}$ & $43.5^{\mathrm{b}}$ & $46.7^{\mathrm{b}}$ & $50.3^{\mathrm{b}}$ & $49.5^{\mathrm{b}}$ & $53.1^{\mathrm{b}}$ & $48.2^{\mathrm{a}}$ & $31.5^{\mathrm{b}}$ & $71.4^{\mathrm{a}, \mathrm{b}, \mathrm{c}}$ & $36.7^{\mathrm{c}}$ & $52.9^{\mathrm{a}}$ & $39.3^{b, c}$ & $49.2^{\mathrm{a}, \mathrm{b}}$ \\
\hline No & 57 & $70.7^{\mathrm{a}}$ & $56.5^{\mathrm{b}}$ & $53.3^{\mathrm{b}}$ & $49.7^{\mathrm{b}}$ & $50.5^{\mathrm{b}}$ & $46.9^{\mathrm{b}}$ & $51.8^{\mathrm{a}}$ & $68.5^{\mathrm{b}}$ & $28.6^{\mathrm{a}, \mathrm{b}, \mathrm{c}}$ & $63.3^{\mathrm{c}}$ & $47.1^{\mathrm{a}}$ & $60.7^{\mathrm{b}, \mathrm{c}}$ & $50.8^{\mathrm{a}, \mathrm{b}}$ \\
\hline \multicolumn{15}{|c|}{ Do you purchase fat reduced food? } \\
\hline Yes & 64 & $52.7^{\mathrm{a}}$ & $62.3^{\mathrm{a}}$ & $67.8^{\mathrm{a}, \mathrm{b}}$ & $67.2^{\mathrm{a}, \mathrm{b}}$ & $78.5^{\mathrm{b}}$ & $75.5^{\mathrm{a}, \mathrm{b}}$ & $70.3^{\mathrm{a}}$ & $49.7^{\mathrm{b}}$ & $71.4^{\mathrm{a}, \mathrm{b}}$ & $58.2^{\mathrm{b}}$ & $71.7^{\mathrm{a}}$ & $65.5^{\mathrm{a}, \mathrm{b}}$ & $66.5^{\mathrm{a}, \mathrm{b}}$ \\
\hline No & 36 & $47.3^{\mathrm{a}}$ & $37.7^{\mathrm{a}}$ & $32.2^{\mathrm{a}, \mathrm{b}}$ & $32.7^{\mathrm{a}, \mathrm{b}}$ & $21.5^{\mathrm{b}}$ & $24.5^{\mathrm{a}, \mathrm{b}}$ & $29.7^{\mathrm{a}}$ & $50.3^{\mathrm{b}}$ & $28.6^{\mathrm{a}, \mathrm{b}}$ & $41.8^{\mathrm{b}}$ & $28.3^{\mathrm{a}}$ & $34.5^{\mathrm{a}, \mathrm{b}}$ & $33.5^{\mathrm{a}, \mathrm{b}}$ \\
\hline \multicolumn{15}{|c|}{ Would you eat salt and fat reduced white and black pudding sausages? } \\
\hline $\begin{array}{c}\text { Yes, if the taste is } \\
\text { similar to conventional } \\
\text { white and black } \\
\text { pudding sausages }\end{array}$ & 50 & $49.8^{\mathrm{a}}$ & $50.1^{\mathrm{a}}$ & $49.3^{\mathrm{a}}$ & $51.5^{\mathrm{a}}$ & $52.3^{\mathrm{a}}$ & $46.9^{\mathrm{a}}$ & $50.5^{\mathrm{a}}$ & $49.7^{\mathrm{a}}$ & $0.0^{\mathrm{b}}$ & $49.6^{\mathrm{a}, \mathrm{b}}$ & $57.0^{\mathrm{a}}$ & $49.6^{\mathrm{a}, \mathrm{b}}$ & $45.3^{\mathrm{a}, \mathrm{b}}$ \\
\hline $\begin{array}{l}\text { Yes, even the taste is } \\
\text { different to } \\
\text { conventional white and } \\
\text { black pudding sausages }\end{array}$ & 15 & $18.5^{\mathrm{a}}$ & $12.7^{\mathrm{a}}$ & $14.5^{\mathrm{a}}$ & $11.7^{\mathrm{a}}$ & $16.8^{\mathrm{a}}$ & $18.4^{\mathrm{a}}$ & $14.3^{\mathrm{a}}$ & $15.5^{\mathrm{a}}$ & $14.3^{\mathrm{a}}$ & $15.6^{\mathrm{a}}$ & $12.3^{\mathrm{a}}$ & $15.0^{\mathrm{a}}$ & $15.1^{\mathrm{a}}$ \\
\hline Not at all & 35 & $31.7^{\mathrm{a}}$ & $37.1^{\mathrm{a}}$ & $36.2^{\mathrm{a}}$ & $36.8^{\mathrm{a}}$ & $30.8^{\mathrm{a}}$ & $34.7^{\mathrm{a}}$ & $35.2^{\mathrm{a}}$ & $34.8^{\mathrm{a}}$ & $85.7^{\mathrm{b}}$ & $34.7^{\mathrm{a}, \mathrm{b}}$ & $30.7^{\mathrm{a}}$ & $35.4^{\mathrm{a}, \mathrm{b}}$ & $39.6^{\mathrm{a}, \mathrm{b}}$ \\
\hline \multicolumn{15}{|c|}{ Would you purchase salt and fat reduced food even when the product is more expensive? } \\
\hline Yes & 56 & $41.5^{\mathrm{a}}$ & $47.9^{\mathrm{a}}$ & $66.4^{\mathrm{b}}$ & $63.7^{\mathrm{b}}$ & $71.0^{\mathrm{b}}$ & $83.7^{\mathrm{b}}$ & $59.0^{\mathrm{a}}$ & $48.7^{\mathrm{b}}$ & $71.4^{\mathrm{a}, \mathrm{b}}$ & $42.1^{\mathrm{b}}$ & $63.9^{\mathrm{a}}$ & $64.1^{\mathrm{a}}$ & $66.5^{\mathrm{a}}$ \\
\hline No & 44 & $58.5^{\mathrm{a}}$ & $52.1^{\mathrm{a}}$ & $33.6^{\mathrm{b}}$ & $36.3^{\mathrm{b}}$ & $29.0^{\mathrm{b}}$ & $16.3^{\mathrm{b}}$ & $41.0^{\mathrm{a}}$ & $51.3^{\mathrm{b}}$ & $28.6^{\mathrm{a}, \mathrm{b}}$ & $57.9^{\mathrm{b}}$ & $36.1^{\mathrm{a}}$ & $35.9^{\mathrm{a}}$ & $33.5^{\mathrm{a}}$ \\
\hline \multicolumn{15}{|c|}{ If yes, how much more money in percentage would you spend on salt and fat reduced food? } \\
\hline $5 \%-15 \%$ & 33 & $23.9^{\mathrm{a}}$ & $26.3^{\mathrm{b}}$ & $44.7^{\mathrm{b}}$ & $39.8^{\mathrm{b}}$ & $42.1^{\mathrm{b}}$ & $42.9^{\mathrm{a}, \mathrm{b}}$ & $34.9^{\mathrm{a}}$ & $28.8^{\mathrm{a}}$ & $42.9^{\mathrm{a}, \mathrm{b}}$ & $24.0^{\mathrm{b}}$ & $39.8^{\mathrm{a}}$ & $41.3^{\mathrm{a}}$ & $35.2^{\mathrm{a}}$ \\
\hline $15 \%-30 \%$ & 16 & $11.2^{\mathrm{a}}$ & $15.8^{\mathrm{a}}$ & $14.5^{\mathrm{a}}$ & $18.7^{\mathrm{a}}$ & $21.5^{\mathrm{a}}$ & $20.4^{\mathrm{a}}$ & $17.0^{\mathrm{a}}$ & $13.6^{\mathrm{a}}$ & $14.3^{\mathrm{a}}$ & $11.7^{\mathrm{a}}$ & $18.9^{\mathrm{a}}$ & $17.5^{\mathrm{a}}$ & $20.1^{\mathrm{a}}$ \\
\hline $30 \%-50 \%$ & 3 & $3.4^{\mathrm{a}}$ & $1.7^{\mathrm{a}}$ & $3.9^{\mathrm{a}}$ & $3.5^{\mathrm{a}}$ & $2.8^{\mathrm{a}}$ & $8.2^{\mathrm{a}}$ & $3.0^{\mathrm{a}}$ & $3.3^{\mathrm{a}}$ & $0.0^{\mathrm{a}}$ & $2.7^{\mathrm{a}}$ & $2.5^{\mathrm{a}}$ & $3.4^{\mathrm{a}}$ & $4.5^{\mathrm{a}}$ \\
\hline $50 \%-75 \%$ & 2 & $0.5^{\mathrm{a}}$ & $3.0^{\mathrm{a}}$ & $2.0^{\mathrm{a}}$ & $1.2^{\mathrm{a}}$ & $0.9^{\mathrm{a}}$ & $0.0^{\mathrm{a}}$ & $2.4^{\mathrm{a}}$ & $0.0^{\mathrm{a}}$ & $14.3^{\mathrm{b}}$ & $2.0^{\mathrm{a}, \mathrm{b}}$ & $1.6^{\mathrm{a}, \mathrm{b}}$ & $0.5^{\mathrm{a}}$ & $2.2^{\mathrm{a}, \mathrm{b}}$ \\
\hline $75 \%-100 \%$ & 1 & $1.0^{\mathrm{a}}$ & $0.8^{\mathrm{a}}$ & $0.7^{\mathrm{a}, \mathrm{b}}$ & $0.6^{\mathrm{a}}$ & $1.9^{\mathrm{a}, \mathrm{b}}$ & $8.2^{\mathrm{b}}$ & $1.0^{\mathrm{a}}$ & $2.0^{\mathrm{a}}$ & $0.0^{\mathrm{a}}$ & $1.2^{\mathrm{a}}$ & $0.8^{\mathrm{a}}$ & $1.0^{\mathrm{a}}$ & $2.2^{\mathrm{a}}$ \\
\hline$>100 \%$ & 1 & $1.5^{\mathrm{a}}$ & $0.1^{\mathrm{a}}$ & $0.7^{\mathrm{a}}$ & $0.0^{\mathrm{a}}$ & $1.9^{\mathrm{a}}$ & $4.1^{\mathrm{a}}$ & $0.8^{\mathrm{a}}$ & $1.0^{\mathrm{a}}$ & $0.0^{\mathrm{a}}$ & $0.7^{\mathrm{a}}$ & $0.4^{\mathrm{a}}$ & $0.5^{\mathrm{a}}$ & $2.2^{\mathrm{a}}$ \\
\hline
\end{tabular}

a,b,c,Percentages within question and row sharing different letters are significantly different.

participants said they know that high sodium levels in food can cause hypertension, cardiovascular disease and stroke (Table 4). Women were found to be statistically more informed than men. No particular age group and level of education were less aware of sodium intake from foods and the health aspects than the others. The question "Do you know that high saturated fatty acids level in foods 
Table 4. Knowledge and beliefs.

\begin{tabular}{|c|c|c|c|c|c|c|c|c|c|c|c|c|c|c|}
\hline \multirow{2}{*}{$\begin{array}{c}\text { Questionnaire } \\
\mathrm{N}=1045\end{array}$} & \multirow{2}{*}{ Total (\%) } & \multicolumn{6}{|c|}{ Age } & \multicolumn{2}{|c|}{ Gender } & \multicolumn{5}{|c|}{ Education } \\
\hline & & $<20$ & $20-29$ & $30-39$ & $40-49$ & $50-59$ & $\geq 60$ & female & male & $\begin{array}{l}\text { No school } \\
\text { certificate }\end{array}$ & $\begin{array}{c}\text { Leaving } \\
\text { certificate }\end{array}$ & $\begin{array}{c}\text { Primary } \\
\text { degree }+ \\
\text { diploma }\end{array}$ & Post-graduate & $\begin{array}{l}\text { Higher } \\
\text { degree }\end{array}$ \\
\hline \multicolumn{15}{|c|}{ Do you know that high sodium levels in food can cause hypertension, cardiovascular diseases and stroke? } \\
\hline Yes & 92 & $88.8^{\mathrm{a}}$ & $91.4^{\mathrm{a}}$ & $92.8^{\mathrm{a}}$ & $94.7^{\mathrm{a}}$ & $96.3^{\mathrm{a}}$ & $89.8^{\mathrm{a}}$ & $93.9^{\mathrm{a}}$ & $87.4^{\mathrm{b}}$ & $85.7^{\mathrm{a}}$ & $91.0^{\mathrm{a}}$ & $91.0^{\mathrm{a}}$ & $93.7^{\mathrm{a}}$ & $94.4^{\mathrm{a}}$ \\
\hline No & 8 & $11.2^{\mathrm{a}}$ & $8.6^{\mathrm{a}}$ & $7.2^{\mathrm{a}}$ & $5.3^{\mathrm{a}}$ & $3.7^{\mathrm{a}}$ & $10.2^{\mathrm{a}}$ & $6.1^{\mathrm{a}}$ & $12.6^{\mathrm{b}}$ & $14.3^{\mathrm{a}}$ & $9.0^{\mathrm{a}}$ & $9.0^{\mathrm{a}}$ & $6.3^{\mathrm{a}}$ & $5.6^{\mathrm{a}}$ \\
\hline \multicolumn{15}{|c|}{ Do you know that high saturated fatty acids level in food can cause obesity, diabetes and stroke? } \\
\hline Yes & 97 & $98.0^{\mathrm{a}}$ & $96.4^{\mathrm{a}}$ & $97.4^{\mathrm{a}}$ & $98.8^{\mathrm{a}}$ & $99.1^{\mathrm{a}}$ & $91.8^{\mathrm{a}}$ & $98.1^{\mathrm{a}}$ & $95.4^{\mathrm{a}}$ & $85.7^{\mathrm{a}}$ & $97.8^{\mathrm{a}}$ & $98.4^{\mathrm{a}}$ & $96.1^{\mathrm{a}}$ & $96.6^{\mathrm{a}}$ \\
\hline No & 3 & $2.0^{\mathrm{a}}$ & $3.6^{\mathrm{a}}$ & $2.6^{\mathrm{a}}$ & $1.2^{\mathrm{a}}$ & $0.9^{\mathrm{a}}$ & $8.2^{\mathrm{a}}$ & $1.9^{\mathrm{a}}$ & $4.6^{\mathrm{a}}$ & $14.3^{\mathrm{a}}$ & $2.2^{\mathrm{a}}$ & $1.6^{\mathrm{a}}$ & $3.9^{\mathrm{a}}$ & $3.4^{\mathrm{a}}$ \\
\hline Yes & 87 & $85.9^{\mathrm{a}}$ & $86.1^{\mathrm{a}}$ & $86.8^{\mathrm{a}}$ & $87.1^{\mathrm{a}}$ & $93.5^{\mathrm{a}}$ & $91.8^{\mathrm{a}}$ & $89.8^{\mathrm{a}}$ & $81.5^{\mathrm{b}}$ & $85.7^{\mathrm{a}, \mathrm{b}}$ & $84.8^{\mathrm{b}}$ & $89.3^{\mathrm{a}, \mathrm{b}}$ & $93.7^{\mathrm{a}}$ & $83.2^{\mathrm{b}}$ \\
\hline No & 13 & $14.1^{\mathrm{a}}$ & $13.9^{\mathrm{a}}$ & $13.2^{\mathrm{a}}$ & $12.9^{\mathrm{a}}$ & $6.5^{\mathrm{a}}$ & $8.2^{\mathrm{a}}$ & $10.2^{\mathrm{a}}$ & $18.5^{\mathrm{b}}$ & $14.3^{\mathrm{a}, \mathrm{b}}$ & $15.2^{\mathrm{b}}$ & $10.7^{\mathrm{a}, \mathrm{b}}$ & $6.3^{\mathrm{a}}$ & $16.8^{\mathrm{b}}$ \\
\hline \multicolumn{15}{|c|}{ Do you think there is a greater need for salt and fat reduced products on the market? } \\
\hline Yes & 83 & $82.4^{\mathrm{a}}$ & $80.3^{\mathrm{a}}$ & $85.5^{\mathrm{a}}$ & $82.5^{\mathrm{a}}$ & $84.1^{\mathrm{a}}$ & $89.8^{\mathrm{a}}$ & $84.4^{\mathrm{a}}$ & $78.5^{\mathrm{a}}$ & $57.1^{\mathrm{a}}$ & $82.2^{\mathrm{a}}$ & $87.3^{\mathrm{a}}$ & $80.1^{\mathrm{a}}$ & $81.6^{\mathrm{a}}$ \\
\hline No & 17 & $17.6^{\mathrm{a}}$ & $19.7^{\mathrm{a}}$ & $14.5^{\mathrm{a}}$ & $17.5^{\mathrm{a}}$ & $15.9^{\mathrm{a}}$ & $10.2^{\mathrm{a}}$ & $15.6^{\mathrm{a}}$ & $21.5^{\mathrm{a}}$ & $42.9^{\mathrm{a}}$ & $17.8^{\mathrm{a}}$ & $12.7^{\mathrm{a}}$ & $19.9^{\mathrm{a}}$ & $18.4^{\mathrm{a}}$ \\
\hline
\end{tabular}

a,b,c Percentages within question and row sharing different letters are significantly different.

can cause obesity, diabetes and stroke?" responded only a minority (3\%) with "no" (Table 4). No significant differences were found between various age groups, gender and level of education.

Completion of the survey was addressed to participants' beliefs. In total, $87 \%$ of respondents think there is a greater need in informing the population about the impact of sodium and saturated fatty acids in food on body health (Table 4). Furthermore, significantly more women accounted for "yes" than men. No particular age group was found to advocate statistically more or less than the others. Significant differences in age were recorded, though no pattern became apparent. Finally, $83 \%$ of participants believe that there is a greater need for salt and fat reduced products on the market (Table 4). Again, significantly more women voted "yes" than men. No statistical differences in attitudes between the various age groups and level of education were found.

\section{Discussion}

The survey completed 1045 participants, with $71 \%$ female and $29 \%$ male. A similar distribution of gender was also found in the study by Tobin, O'Sullivan, Hamill, \& Kerry (2014) [38]. Thus, it is observed that in these instances females are more likely to complete surveys about consumer attitudes on foods linked with health aspects.

In general, it seems that public health campaigns have succeeded as only $2 \%$ of the participants did not take care at all with the consumption of a balanced diet independent of age, gender and level of education. Following the partici- 
pants' self-assessment, 38\% take a "bit of care" with a balanced diet and 59\% of them pay "complete attention". In the present survey significantly more women than men take complete care of a balanced diet. These findings are consistent to the published work by [58] who surveyed trends in dietary salt intake from processed food sources and discretionary salt among consumers $(n=360)$ in Northern Ireland. Males showed a significantly more frequent consumption of sausages, tinned vegetables, meat pies and chips compared to females. Accordingly, the authors concluded that "men are less aware of good dietary practice and the importance of healthy eating messages" which can be confirmed by the present study. However, in the present study participants aged under 30 take significantly less care of a complete balanced diet. The authors Purdy \& Arrmstrong (2007) [58] stated that participants aged 15 to 29 had a significantly higher consumption of pizza and crisps than those aged 30 and over. Warwick (1998) [59] postulated that snack foods are popular among this age group. However, in the present study, higher educated participants take significantly more care of a complete balanced diet. In contrast, Purdy \& Arrmstrong (2007) [58] reported that the higher educated participants consumed pizza and ready meals more frequently. It was suggested that these consumers have less time to prepare meals and consequently they purchase far more convenient foods. In the present study, more than half of the participants (59\%) maintained a balanced diet. Therefore, it is important to shift consumers who stated they take just a bit care of maintaining a balanced to an exclusively balanced diet with continuing messages on healthy food consumption. Hence, it is even more important that food manufacturers produce foods low in salt and saturated fatty acids that are also convenient.

Since the WHO first promoted the introduction of foods low in salt and fat, an increased number of food producers have taken the challenge to manufacture sensory acceptable salt and saturated fatty acids reduced foods. In the present study significantly more women than men, those with "diploma and primary" degree than individuals with a "school leaving certificate" and with increased age voted to purchase salt and fat reduced food. In general, consumers purchased more fat reduced (64\%) than salt reduced foods (43\%). Although only less than half of the participants consume salt reduced foods, it is already twice as much it was in the study by Purdy \& Arrmstrong (2007) [58] with 19\%. Therefore, the result in the present study shows that fat reduced foods are already well accepted and an increase in purchasing salt reduced foods was achieved within the last few years. In the present study most of the consumers ( $>90 \%)$ were aware of the health risks leading by a diet rich in salt and fat. Consequently, the lower acceptance for salt reduced foods (in comparison to fat reduced foods) may lie in the fact that consumers are not satisfied with the current product range on the market rather than any lack of consumers' knowledge or awareness. The product development of fat reduced foods is already far more developed than for salt reduced foods. Considering the increased purchase rate of salt reduced foods from 2007 with $19 \%$ [58] to the present with $43 \%$ (present study), it seems that the 
food industry are already successfully addressing these difficulties with reformulating salt reduced products.

However, the author Jordana (2000) [42] stated that traditional foods show an insufficient adaption to new requirements. To clarify if consumers see a conflict between innovation and concept of traditional foods, in the present survey participants were asked if they would eat salt and fat reduced white and black puddings. It is important to note that white and black puddings have a very high value for the Irish and British consumer. Independent of age, gender and level of education in total $65 \%$ of participants would eat salt and fat reduced white and black puddings, and among 15\% of participants would eat it even the taste is different to conventional white and black puddings. Similar ratings were observed for purchasing fat reduced conventional foods and even lower acceptance were recorded for purchasing salt reduced conventional foods. Therefore, based on this result the consumers do not distinguish between traditional foods and conventional foods when it comes to salt and fat reduction. In the present survey only a minority (15\%) of the participants were willing to purchase salt and fat reduced white and black pudding and tolerate differences in taste which again, confirms the conclusion of Guerrero et al. (2009) [60]. that innovations in traditional food products were only accepted by consumers if they provide manifest benefits like safety or health improvements. Ronteltap, van Trijp, Renes, \& Frewer (2007) [61] stated that besides perceiving benefits also costs are a major determinant of consumers acceptance of food innovations. In general, higher costs for salt and fat reduced foods can be generally expected as salt and fat reductions are achievable by stealth through increasing other ingredients (higher in quality), using replacers/enhancer and by compensation the lack of taste with flavour delivery agents (e.g. herbs, spices, lemon, onion/garlic, vinegar, etc.) and losses in yeild [62] [63]. It was estimated that a sodium reduction of $20 \%$ to $30 \%$ results in food cost increases of $5 \%$ to $30 \%$ depending on the type of food product [64]. In the present study two-thirds of the participants spend between 15\% and $30 \%$ of their income on food. Slightly more than half of participants were willing to pay more money for salt and fat reduced foods. Thirty-three percent of all participants would accept food cost increases of 5\% to $15 \%$, which still is not fully covering the cost increases for all types of food estimated by Dötsch et al. (2009) [64]. Sixteen percent of all participants tolerate increases of $15 \%$ to $30 \%$ and the minority (7\%) would even pay more than $30 \%$ for salt and fat reduced foods. Therefore, only roughly a quarter of all participants are willing to pay the actual estimated cost increase.

Participants in the age groups $<20$ and 20 to 29 reached the highest rates (53\% and 58\%) in that they would like to spend more money on foods if more money was available, and in turn the lowest rates $(42 \%$ and $48 \%)$ in that they would purchase more expensive salt and fat reduced foods. The inverse effect was observed for the age groups 50 to 59 and over 59 . Among, $71 \%$ and accordingly $84 \%$ of them would purchase salt and fat reduced foods even it was more 
expensive. Only $33 \%$ and $18 \%$ respectively would like to spend more money on foods, if more money was available. A similar pattern was also found for the different grades of education. Apparently the reason that younger participants $(<$ 30 years) and lower educated participants are less willing to pay more money for salt and fat reduced foods might be simply due to their financial situation. People with professional occupations generally have higher disposable incomes which in turn can be spent more on groceries [65]. Additionally, elderly people are usually already more often confronted with civilisation diseases whereby their diet might be under medical supervision, and hence they have a higher willingness in spending more money on salt and fat reduced food. Furthermore, significantly more women are willing to spend more money on salt and fat reduced foods than men, although more women than men would like to spend more money on foods in general, if they would have more money available (not significant). Women, in general, showed a higher willingness for purchasing salt and fat reduced foods than men. The higher degree of willingness can be explained by the fact that most of the food shopping is completed by women [66] and additionally, significantly more women were aware of the health risks lead by a diet rich in salt.

However, messages on health risks associated with a diet rich in salt and saturated fatty acids seem to have reached the participants (92\% and $97 \%$ respectively), independent of age and educational status. Men were significantly less aware of the consequences of a diet rich in salt (than women). However, in comparison to consumers' knowledge, less participants take, in general, complete care of a maintaining a balanced diet (59\%) and consume already salt (43\%) and fat (64\%) reduced foods. Despite the participants being very well informed, obviously less participants transferred this knowledge into their dietary intakes. However, in the previous study by Purdy and Armstong (2007) [58] consumers of lower-economic status were less aware of the link between health and diet, which cannot be confirmed. Therefore, public health campaigns have raised consumers' awareness more comprehensively. Nevertheless, the majority (87\%) of the participants thinks that there is still a greater need in informing people about the impact of sodium and saturated fatty acids in foods on body health. Independent of age, gender and level of education, $83 \%$ of the participants think that there is a greater need for salt and fat reduced food products on the market. Therefore, the majority of respondents are informed and looking for more information and a wider range of salt and fat reduced foods. The fewer acceptances might be caused by the relative small current product range on the market, especially of salt reduced foods, and the higher prices they are sold at.

To counteract higher prices for low salt and low fat foods, taxation of food high in salt, fat (and sugar) might be useful. Taxation of unhealthy foods and beverages are already controversially discussed. The authors Duffey \& Gordon-Larsen (2010) [67] evidenced that food consumption is linked with food prices which has a direct influence on body health. Consequently, taxation may influence consumers 
inequality [68]. However, launching taxation and subsidies of diverse foods and beverages might additional underline the importance of a healthy diet and guide all consumers in distinguishing products high and low in salt, fat and sugar, independent of consumers' disposable income. This is very topical in Ireland currently due to the imminent implementation of a sugar tax on beverages in 2018 .

Denmark, introduced in 2011, the world's first saturated fat tax. The study by Bødker, Pisinger, Toft, \& Jørgensen (2015) [69] revealed that with the launch of the fat tax the food industry representatives fought against it with special tactics such as threatening lawsuits, predicting a damage to Denmark's economy, casting doubts on the link between saturated fatty acids and health risks, diverting focus to other food product categories and requesting postponements. Additionally, the fat tax suffered from significant shortcomings (e.g. tax did not reflect the fat content in the final product) and gradually suffered a loss of popularity among politicians, health experts and public. Consequently, 15 months later the fat tax was abolished, even when a lower consumption of saturated fat was beginning to appear.

Additionally to taxation/subsidies, user-friendly product labelling schemes are generally required as not all consumers read product labels or understand them. Through the EU-Regulation 1169/2011 (article 9 (1) 1), declaration of nutrition is a mandatory legislation since $13^{\text {th }}$ December 2014 . However, an exclusive listing of nutritional values might not be enough for making an informed purchase decision by consumers. Using reference intakes (RI) labels, the previously used term "guideline daily amount" (GDA), might help consumers to evaluate more easily how a particular food product fits into their daily diet, and furthermore to manage their key nutrients (fat, saturated fat, salt, sugars) and calorie intakes. Although, RI values are applied for adults, they are based on the requirements for an average healthy individual with no special dietary requirements (sport activities, special diet, pregnant and breast feeding mothers) with a calculated energy intake of $2000 \mathrm{kcal}$. Currently, declaration of RI is optional (EU-Regulation 1169/2011, article $32(4))$.

\section{Conclusion}

In Ireland, it seems that public health campaigns relating the importance of a balanced diet generally reached the consumer, as only a minority of the participants did not take care at all about maintaining a balanced diet, independent of age, gender and level of education. It was observed that $59 \%$ of participants take complete care in maintaining a balanced diet and $38 \%$ of participants take at least a bit of care. It is now important to shift these consumers' group attitudes to actively maintaining a fully balanced diet. Furthermore, an increase in purchasing salt reduced food (43\%) was observed. Fat reduced food products were already better accepted (64\%). In general, men, young adults ( $<30$ years) and lower educated people purchased less salt and fat reduced foods and took less care of a balanced diet. Based on the results of the present study no conflict can 
be seen for traditional foods reduced in salt and fat content when intrinsic character is kept. However, the majority of the participants (>90\%) were aware of the health risks leading from a diet rich in salt and fat, although less participants transferred this knowledge into their diet. A very high demand for more salt and fat reduced foods on the market was observed although smaller product ranges and higher prices might diminish the level of acceptance. Launching taxation and subsidies in tandem might counteract this. On the one hand, food manufacturers might be encouraged in reformulating their products, and on the other hand, it underlines to consumers the importance of a healthy diet. Furthermore, a user-friendly product labelling scheme is required so that consumers are able to make easier food choices.

\section{References}

[1] Hitchman, C., Christie, I., Harrison, M. and Lang, T., Eds. (2002) Inconvenience Food. The Struggle to Eat Well on a Low Income. London.

[2] Henderson, L., Irving, K., Gregory, J., Bates, C., Prentice, A., Perks, J. and Farron, M. (2003) National Diet \& Nutrition Survey: Adults aged 19 to 64.

[3] Eaton, S. and Konner, M. (1985) Paleolithic Nutrition. A Consideration of Its Nature and Current Implications. New England Journal of Medicine, 312, 283-289. https://doi.org/10.1056/NEJM198501313120505

[4] Lewington, S., Clarke, R., Qizilbash, N., Peto, R. and Collins, R. (2002) Age-Specific Relevance of Usual Blood Pressure to Vascular Mortality: A Meta-Analysis of Individual Data for One Million Adults in 61 Prospective Studies. Lancet, 360, 1903-1913. https://doi.org/10.1016/S0140-6736(02)11911-8

[5] WHO (2000) Obesity: Preventing and Managing the Global Epidemic. Geneva.

[6] Zock, P. (2006) Health Problems Associated with Saturated and Trans Fatty Acids Intake. In: Williams, C. and Buttriss, J., Eds., Improving the Fat Content of Foods, Woodhead Publishing Ltd., Cambridge, 3-24. https://doi.org/10.1533/9781845691073.1.3

[7] Mennell, S., Murcott, A. and Van Otterloo, A. (1993) The Sociology of Food, Eating, Diet and Culture. 2nd Edition, SAGE Publications Ltd.

[8] James, W., Nelson, M., Ralph, A. and Leather, S. (1997) Socio-Economic Determinants of Health: The Contribution of Nutrition to Inequalities in Health. British Medical Journal, 314, 1545-1550. https://doi.org/10.1136/bmj.314.7093.1545

[9] WHO (2012) Guideline: Sodium Intake for Adults and Children. http://apps.who.int/iris/handle/10665/77985

[10] WHO (2003) Diet, Nutrtition and the Prevention of Chronic Diseases.

[11] Lindsay, R. (2007) Flavours. In: Damodaran, S., Parkin, K.L. and Fennema, O.R., Eds., Fennema's Food Chemistry, 4th Edition, Taylor and Francis Group.

[12] Fellendorf, S., O’Sullivan, M.G. and Kerry J.P. (2016) Effect of Using Replacers on the Physicochemical Properties and Sensory Quality of Low Salt and Low Fat White Puddings. European Food Research and Technology, 242, 2105-2118. https://doi.org/10.1007/s00217-016-2707-z

[13] McClements, D.J. (2004) Food Emulsions: Principles, Practice and Techniques. 2nd Edition, CRC Press, Boca Raton. https://doi.org/10.1201/9781420039436

[14] Giasson, S., Israelachvili, J. and Yoshizawa, H. (1997) Thin Film Morphology and 
Tribology Study of Mayonnaise. Journal of Food Science, 62, 640. https://doi.org/10.1111/j.1365-2621.1997.tb15427.x

[15] Hansch, C., Leo, A.J. and Hoekman, D. (1995) Exploring QSAR. Hydrophobic, Electronic, and Steric Constants. American Chemical Society, Washington DC.

[16] Richardson, A.M., Tyuftin, A.A., Kilcawley, K.N., Gallagher, E., O’Sullivan, M.G. and Kerry, J.P. (2018) The Impact of Sugar Particle Size Manipulation on the Physical and Sensory Properties of Chocolate Brownies. LWT-Food Science and Technology, 95, 51-57. https://doi.org/10.1016/j.lwt.2018.04.038

[17] Henneberry, S., O’Sullivan, M.G., Kilcawley, K.N. Kelly, P.M., Wilkinson, M.G. and Guinee, T.P. (2016) Sensory Quality of Unheated and Heated Mozzarella-Style Cheeses with Different Fat, Salt and Calcium Levels. International Journal of Dairy Science, 69, 38-50. https://doi.org/10.1111/1471-0307.12300

[18] O’Sullivan, M.G. (2017) CH9. Nutritionally Optimised Low Fat Foods. In: $A$ Handbook for Sensory and Consumer Driven New Product Development. Innovative Technologies for the Food and Beverage Industry, Woodhead Publishing Ltd., UK, 177-196. https://doi.org/10.1016/B978-0-08-100352-7.00009-9

[19] O’Sullivan, M.G. (2017) CH10. Sensory and Consumer Led Innovative Product Development-From Inception to the Shelf (Current and Future Methodologies). In: A Handbook for Sensory and Consumer Driven New Product Development. Innovative Technologies for the Food and Beverage Industry, Woodhead Publishing Ltd., UK, 197-222. https://doi.org/10.1016/B978-0-08-100352-7.00010-5

[20] O’Sullivan, M.G. (2017) CH11. Sensory Properties Affecting Meat and Poultry Quality. In: A Handbook for Sensory and Consumer Driven New Product Development. Innovative Technologies for the Food and Beverage Industry, Woodhead Publishing Ltd., UK, 225-257. https://doi.org/10.1016/B978-0-08-100352-7.00011-7

[21] O’Sullivan, M.G. (2017) CH12. Sensory Properties of Dairy products. In: A Handbook for Sensory and Consumer Driven New Product Development. Innovative Technologies for the Food and Beverage Industry, Woodhead Publishing Ltd., UK, 259-280. https://doi.org/10.1016/B978-0-08-100352-7.00012-9

[22] O’Sullivan, M.G. (2017) CH13. Sensory Properties of Beverage Products. In: A Handbook for Sensory and Consumer Driven New Product Development. Innovative Technologies for the Food and Beverage Industry, Woodhead Publishing Ltd., UK, 281-304. https://doi.org/10.1016/B978-0-08-100352-7.00013-0

[23] O’Sullivan, M.G. (2017) CH14. Sensory Properties of Bakery and Confectionary Products. In: A Handbook for Sensory and Consumer Driven New Product Development. Innovative Technologies for the Food and Beverage Industry, Woodhead Publishing Ltd., UK, 305-324. https://doi.org/10.1016/B978-0-08-100352-7.00014-2

[24] Conroy, P., O’Sullivan, M.G, Hamill, R.H. and Kerry, J.P. (2017) Sensory Capabilities of Young, Middle Aged and Elderly Irish Assessors to Identify Beef Steaks of Varying Texture. Meat Science, 132, 125-130. https://doi.org/10.1016/j.meatsci.2017.05.020

[25] Conroy, P.M., O’Sullivan, M.G, Hamill, R.H. and Kerry, J.P. (2018) Impact on the Physicochemical and Sensory Properties of Salt- and Fat-Reduced Traditional Irish Breakfast Sausages on Various Age Cohorts Acceptance. Meat Science, 143, 190-198. https://doi.org/10.1016/j.meatsci.2018.04.037

[26] Aaslyng, M.D., Vestergaard, C. and Koch, A.G. (2014) The Effect of Salt Reduction on Sensory Quality and Microbial Growth in Hotdog Sausages, Bacon, Ham and Salami. Meat Science, 96, 47-55. https://doi.org/10.1016/j.meatsci.2013.06.004

[27] Aliño, M., Grau, R., Toldrá, F. and Barat, J.M. (2010) Physicochemical Changes in 
Dry-Cured Hams Salted with Potassium, Calcium and Magnesium Chloride as a Partial Replacement for Sodium Chloride. Meat Science, 86, 331-336. https://doi.org/10.1016/j.meatsci.2010.05.003

[28] Aliño, M., Grau, R., Toldrá, F., Blesa, E., Pagán, M.J. and Barat, J.M. (2010) Physicochemical Properties and Microbiology of Dry-Cured Loins Obtained by Partial Sodium Replacement with Potassium, Calcium and Magnesium. Meat Science, 85, 580-588. https://doi.org/10.1016/j.meatsci.2010.03.009

[29] Fellendorf, S., O’Sullivan, M.G. and Kerry, J.P. (2015) Impact of Varying Salt and Fat Levels on the Physiochemical Properties and Sensory Quality of White Pudding Sausages. Meat Science, 103, 75-82. https://doi.org/10.1016/j.meatsci.2014.12.010

[30] Fellendorf, S., O’Sullivan, M.G. and Kerry, J.P. (2017) Effect of Different Salt and Fat Levels on the Physicochemical Properties and Sensory Quality of Black Pudding. Food Science \& Nutrition, 5, 273-284. https://doi.org/10.1002/fsn3.390

[31] Fellendorf, S., O’Sullivan, M.G. and Kerry, J.P. (2016) Impact of Using Replacers on the Physicochemical Properties and Sensory Quality of Reduced Salt and fat black Pudding. Meat Science, 113, 17-25. https://doi.org/10.1016/j.meatsci.2015.11.006

[32] Fellendorf, S., Kerry, J.P., Hamill, R.H. and O'Sullivan, M.G. (2018) Impact on the Physicochemical and Sensory Properties of Salt Reduced Corned Beef Formulated with and without the Use of Salt Replacers. LWT-Food Science and Technology, 92, 584-592. https://doi.org/10.1016/j.lwt.2018.03.001

[33] Gou, P., Guerrero, L., Gelabert, J. and Arnau, J. (1996) Potassium Chloride, Potassium Lactate and Glycine as Sodium Chloride Substitutes in Fermented Sausages and in Dry-Cured Pork Loin. Meat Science, 42, 37-48.

https://doi.org/10.1016/0309-1740(95)00017-8

[34] Guàrdia, M.D., Guerrero, L., Gelabert, J., Gou, P. and Arnau, J. (2008) Sensory Characterisation and Consumer Acceptability of Small Calibre Fermented Sausages with $50 \%$ Substitution of $\mathrm{NaCl}$ by Mixtures of $\mathrm{KCl}$ and Potassium Lactate. Meat Science, 80, 1225-1230. https://doi.org/10.1016/j.meatsci.2008.05.031

[35] Tobin, B.D., O’Sullivan, M.G., Hamill, R.M. and Kerry, J.P. (2012) Effect of Varying Salt and Fat Levels on the Sensory Quality of Beef Patties. Meat Science, 4, 460-465. https://doi.org/10.1016/j.meatsci.2012.02.032

[36] Tobin, B.D., O’Sullivan, M.G., Hamill, R.M. and Kerry, J.P. (2012) Effect of Varying Salt and Fat Levels on the Sensory and Physiochemical Quality of Frankfurters. Meat Science, 92, 659-666. https://doi.org/10.1016/j.meatsci.2012.06.017

[37] Tobin, B.D., O’Sullivan, M.G., Hamill, R.M. and Kerry, J.P. (2013) The Impact of Salt and Fat Level Variation on the Physiochemical Properties and Sensory Quality of Pork Breakfast Sausages. Meat Science, 93, 145-152. https://doi.org/10.1016/j.meatsci.2012.08.008

[38] Tobin, B.D., O’Sullivan, M.G., Hamill, R.M. and Kerry, J.P. (2013) European Consumer Attitudes on the Associated Health Benefits of Neutraceutical-Containing Processed Meats Using Co-Enzyme Q10 as a Sample Functional Ingredient. Meat Science, 97, 207-213. https://doi.org/10.1016/j.meatsci.2014.01.010

[39] Tobin, B.D., O’Sullivan, M.G., Hamill, R.M. and Kerry, J.P. (2014) Effect of Cooking and in Vitro Digestion on Co-Enzyme Q10 in Processed Meat Products Fortified with Co-Enzyme Q10. Food Chemistry, 150, 187-192.

https://doi.org/10.1016/j.foodchem.2013.10.138

[40] Delgado-Pando, G., Allen, P., Fischer, E., O’Sullivan, M., Kerry, J. and Hamill, R.M. (2018) Salt Content and Minimum Acceptable Levels in Whole-Muscle Cured Meat Products. Meat Science, 139, 179-186. https://doi.org/10.1016/j.meatsci.2018.01.025 
[41] Delgado-Pando, G., Allen, P., O'Sullivan, M., Kerry, J. and Hamill, R.M. (2018) Development of Optimised Reduced-Salt Streaky Rashers through Modification of Formulation and Processing. Meat Science, submitted.

[42] Jordana, J. (2000) Traditional Foods: Challenges Facing the European Food Industry. Food Research International, 33, 147-152. https://doi.org/10.1016/S0963-9969(00)00028-4

[43] Kilcawley, K.N., Faulkner, H., Clarke, H.J., O’Sullivan, M.G. and Kerry, J.P. (2018) Factors Influencing the Flavor of Bovine Milk and Cheese from Grass Based versus Non-Grass Based Milk Production Systems. Foods, 7, 37. https://doi.org/10.3390/foods7030037

[44] Faulkner, H., O’Callaghan, T.F., McAuliffe, S., Hennessy, D., Stanton, C., O'Sullivan, M.G., Kerry, J.P. and Kilcawley, K.N. (2017) Effect of Different Forage Types on the Volatile and Sensory Properties of Bovine Milk. Journal of Dairy Science, 101, 1034-1047. https://doi.org/10.3168/jds.2017-13141

[45] Hickey, C.D., O’Sullivan, M.G., Davis, J., Scholz, D., Kilcawley, K.N., Wilkinson, M.G. and Sheehan, J.J. (2018) The Effect of Buttermilk or Buttermilk Powder Addition on Functionality, Textural, Sensory and Volatile Characteristics of Cheddar-Style Cheese. Food Research International, 103, 468-477. https://doi.org/10.1016/j.foodres.2017.09.081

[46] O’Callaghan, T., Mannion, D.T., Hennessy, D., McAuliffe, S., O’Sullivan, M.G., Leeuwebdaal, N., Beresford, T.P., Dillon, P., Kilcawley, K.N., Sheehan, D.J., Ross, P. and Stanton, C. (2017) Effect of Pasture versus Indoor Feeding Systems on Quality Characteristics, Nutritional Composition, and Sensory and Volatile Properties of Full-Fat Cheddar Cheese. Journal of Dairy Science, 100, 6053-6073. https://doi.org/10.3168/jds.2016-12508

[47] O'Callaghan, T., Faulkner, H., McAuliffe, S., O’Sullivan, M.G., Hennessy, D., Dillon, P., Kilcawley, K.N., Stanton, C. and Ross, P. (2016) Quality Characteristics, Chemical Composition, and Sensory Properties of Butter from Cows on Pasture versus Indoor Feeding Systems. Journal of Dairy Science, 99, 9441-9460.

https://doi.org/10.3168/jds.2016-11271

[48] Stokes, C., O’Sullivan, M.G. and Kerry, J.P. (2016) Assessment of Black Coffee Temperature Profiles Consumed from Paper-Based Cups and Effect on Affective and Descriptive Product Sensory Attributes. International Journal of Food Science and Technology, 51, 2041-2048. https://doi.org/10.1111/ijfs.13176

[49] Stokes, C., O’Sullivan, M.G. and Kerry, J.P. (2017) Hedonic and Descriptive Sensory Evaluation for Development of Novel Instant and Fresh Coffee Products. European Food Research and Technology, 243, 331-340. https://doi.org/10.1007/s00217-016-2747-4

[50] Stokes, C., O’Sullivan, M.G. and Kerry, J.P. (2018) Rapid Descriptive Consumer Analysis Using Simultaneous and Monadic Sample Presentation for Coffee. Food and Nutrition Sciences, 9, 63-76. https://doi.org/10.4236/fns.2018.92005

[51] Stefanovic, E., Kilcawley, K.N., Roces, C., Rea, M., O’Sullivan, M.G., Sheehan, J.J. and McAuliffe, O. (2018) Evaluation of the Potential of Lactobacillus paracasei Adjuncts for Flavor Compounds Development and Diversification in Short-Aged Cheddar Cheese. Frontiers in Microbiology, 9, 1506.

https://doi.org/10.3389/fmicb.2018.01506

[52] Walsh, A.M., Crispie, F., Kilcawley, K., O’Sullivan, O., O’Sullivan, M.G., Claesson, M.J. and Cotter, P.D. (2017) Correction for Walsh et al., Microbial Succession and Flavor Production in the Fermented Dairy Beverage Kefir. mSystems, 2, e00003-17.

[53] He, F.J. and MacGregor, G.A. (2007) Dietary Salt, High Blood Pressure and Other 
Harmful Effects on Health. In: Kilcast, D. and Angus, F., Eds., Reducing Salt in Foods: Practical Strategies, Woodhead Publishing Ltd., Cambridge, England, 18-54.

[54] de Barcellos, M.D., Grunert, K.G. and Scholderer, J. (2011) Processed Meat Products: Consumer Trends and Emerging Markets. In: Kerry, J.P. and Kerry, J.F., Eds., Processed Meats. Improving Safety, Nutrition and Quality, Woodhead Publishing Ltd., Cambridge, New Delhi, 30-53.

[55] Prosslow (2016) Development of Consumer Accepted Low Salt and Low Fat Irish Traditional Processed Meats. Project Coordinator: Dr. Maurice O’Sullivan. Ref: 11F026.

http://scitechconnect.elsevier.com/sensory-optimised-salt-fat-reduction-processed$\underline{\text { meats/ }}$

[56] Hochberg, Y. (1988) A Sharper Bonferroni Test for Multiple Tests of Significance. Biometrika, 75, 800-802. https://doi.org/10.1093/biomet/75.4.800

[57] Beasley, T.M. and Schumacker, R.E. (1995) Multiple Regression Approach to Analyzing Contingency Tables: Post Hoc and Planned Comparison Procedures. The Journal of Experimental Education, 64, 79-93. https://doi.org/10.1080/00220973.1995.9943797

[58] Purdy, J. and Arrmstrong, G. (2007) Dietary Salt and the Consumer: Reported Consumption and Awareness of Associated Health Risks-A Consumer Perspective. In: Kilcast, D. and Angus, F., Eds., Reducing Salt in Foods: Practical Strategies, Woodhead Publishing Ltd., Cambridge, England, 106-116. https://doi.org/10.1201/9781439824511.ch5

[59] Warwick, J. (1998) Food Choices of Young People in Northern Ireland-The Influences and Health Implications. University of Ulster, Northern Ireland.

[60] Guerrero, L., Guàrdia, M.D., Xicola, J., Verbeke, W., Vanhonacker, F., ZakowskaBiemans, S. and Hersleth, M. (2009) Consumer-Driven Definition of Traditional Food Products and Innovation in Traditional Foods. A Qualitative Cross-Cultural Study. Appetite, 52, 345-354. https://doi.org/10.1016/j.appet.2008.11.008

[61] Ronteltap, A., van Trijp, J.C.M., Renes, R.J. and Frewer, L.J. (2007) Consumer Acceptance of Technology-Based Food Innovations: Lessons for the Future of Nutrigenomics. Appetite, 49, 1-17. https://doi.org/10.1016/j.appet.2007.02.002

[62] Kilcast, D. and Ridder, C. (2007) Sensory Issues in Reducing Salt in Food Products. In: Kilgast, D. and Angus, F., Eds., Reducing Salt in Foods: Practical Strategies, Woodhead Publishing Ltd., Cambridge, England, 201-220. https://doi.org/10.1533/9781845693046

[63] Talbot, G. (2011) Saturated Fats in Foods and Strategies for their replacement: an Introduction. In: Talbot, G., Ed., Reducing Saturated Fats in Foods, Woodhead Publishing Ltd., Cambridge, New Delhi. https://doi.org/10.1533/9780857092472.1.3

[64] Dötsch, M., Busch, J., Batenburg, M., Liem, G., Tareilus, E. and Müller, G.M. (2009) Strategies to Reduce Sodium Consumption: A Food Industry Perspective. Food Science and Nutrition, 49, 841-851. https://doi.org/10.1080/10408390903044297

[65] Bardsley, N. (2000) Ready Meals: Market Report Plus 2000. 5th Edition, Key Note Publications.

[66] Murcott, A. (2000) Is It Still a Pleasure to Cook for Him? Social Changes in the Household and the Family. Journal of Consumer Studies and Home Economics, 24, 78-84. https://doi.org/10.1046/j.1365-2737.2000.00148.x

[67] Duffey, K. and Gordon-Larsen, P. (2010) Food Price and Diet and Health Outcomes: 20 Years of the CARDIA Study. Archives of Internal Medicine, 170, 420-426. https://doi.org/10.1001/archinternmed.2009.545 
[68] Härkänen, T., Kotakorpi, K., Pietinen, P., Pirttilä, J., Reinivuo, H. and Suoniemi, I. (2014) The Welfare Effects of Health-Based Food Tax Policy. Food Policy, 49, 196-206. https://doi.org/10.1016/j.foodpol.2014.07.001

[69] Bødker, M., Pisinger, C., Toft, U. and Jørgensen, T. (2015) The Rise and Fall of the World's First Fat Tax. Health Policy, 119, 737-742.

https://doi.org/10.1016/j.healthpol.2015.03.003 